IP $\rightleftharpoons$ B

\title{
Influência de impactos antrópicos em fragmentos de Floresta Ombrófila Mista em Santa Catarina
}

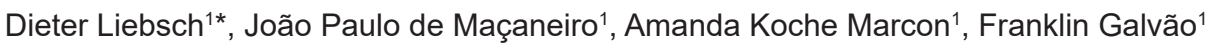 \\ Universidade Federal do Paraná, Av. Prefeito Lothário Meissner, 900, CEP 80210-170, Curitiba, PR, Brasil
}

*Autor correspondente:

dieterliebsch@yahoo.com.br

Termos para indexação:

Corte seletivo

Araucaria angustifolia

Ocotea porosa

Index terms:

Selective felling

Araucaria angustifolia

Ocotea porosa

Histórico do artigo:

Recebido em 05/04/2016

Aprovado em 31/08/2016

Publicado em 30/09/2016

doi: 10.4336/2016.pfb.36.87.1213
Resumo - Em toda região da Floresta Ombrófila Mista, parte do Bioma Mata Atlântica, o corte seletivo e pastoreio são atividades muito comuns. Com o objetivo de relacionar a composição e estrutura com o histórico de uso, foram avaliados os indivíduos arbóreos adultos e juvenis de 12 fragmentos em Santa Catarina. Os fragmentos com menor impacto antrópico apresentaram, para o estrato adulto, maior riqueza e amplitude diamétrica, e menor densidade e frequência de múltiplos fustes, apesar da área basal não diferir entre os fragmentos. Quanto ao estrato juvenil, os fragmentos com maior impacto apresentaram maior riqueza e menor densidade. Os resultados demonstram que o impacto antrópico sobre a comunidade arbórea foi de tal magnitude, que, mesmo passadas algumas décadas, diferenças florísticas e estruturais permanecem evidentes, com tendência a se manterem por longo período.

\section{Influence of anthropic impacts on remnants of Ombrophilous Mixed Forest in Santa Catarina State, Brazil}

\begin{abstract}
Selective cutting and pastures are common activities in the Ombrophilous Mixed Forest, which is part of the Atlantic Rain Forest Biome. Our goal was to relate floristic composition and structure unit with its past use. Adult and juvenile wood species of twelve forest fragments were evaluated in Santa Catarina State. Fragments with less anthropic impacts showed greater richness and diameter range, and lower density and frequency of multiple stems in the adult stratum, although basal area did not differ among fragments. Fragments with higher historical impact showed greater richness and lower density of juvenile stratum. The results showed that human impact on wood species community was so great that even after some decades floristic composition and structure differences remain evident and might remain over a long period.
\end{abstract}

\section{Introdução}

Florestas tropicais e subtropicais estão sujeitas a distúrbios de diversas naturezas, intensidades e frequências. Entre os distúrbios de origem natural, estão associados eventos climáticos extremos, os quais podem ocasionar queda de árvores, abertura de clareiras e movimentação de solo (Chazdon, 2003). Os distúrbios antrópicos podem ser aqueles relacionados à completa remoção das florestas (Fearnside, 2006), ou ao corte seletivo de espécies com valor econômico (OsazuwaPeters et al., 2015).
A retirada de espécies com valor econômico é uma prática muito comum no Brasil. Atualmente, essa atividade é regulamentada e incentivada pelo governo federal como forma de manter as florestas e gerar recursos para sua proteção, sobretudo no domínio Amazônico (Karsten et al., 2013). Por outro lado, no domínio da Mata Atlântica é ilegal, com base na Lei $n^{\circ}$ 11.428 (Brasil, 2006), embora tenha sido uma prática habitual nos séculos passados e responsável por grande parte da atividade econômica do período (Souza et al., 2012). 
Após a alteração no ecossistema, a velocidade com que o ambiente responde é dependente da intensidade e tipo de distúrbio, fazendo com que a floresta apresente diferentes trajetórias sucessionais (Tabarelli \& Mantovani, 1999; Pereira et al., 2015). São observadas modificações em termos de riqueza de espécies, densidade de indivíduos, área basal e distribuição diamétrica, susceptibilidade ao fogo e vento, colonização de espécies de taquaras e bambus, mortalidade dos indivíduos remanescentes, entre outros (Tabarelli \& Mantovani, 1999; Chazdon, 2003; Osazuwa-Peters et al., 2015; Pereira et al., 2015).

A Floresta Ombrófila Mista, também conhecida por Floresta com Araucária, é uma das fitofisionomias vegetacionais inseridas no domínio da Mata Atlântica e predominante no planalto meridional da região Sul do Brasil, além de ser considerada como um hotspot para a conservação da biodiversidade (IBGE, 2012). Compilações de dados recentes indicaram a presença de 925 a 946 espécies espermatófitas nesta fitofisionomia (Gasper et al., 2013; Duarte et al., 2014). A FOM está sujeita a estacionalidade climática, o que ocasiona períodos com baixas temperaturas e ocorrência de geadas, limitando a expansão geográfica de muitas espécies (Duarte et al., 2014).

Atualmente, estima-se que a Floresta Ombrófila Mista mantém uma cobertura remanescente de apenas 12,3\% (Ribeiro et al., 2009), sendo que para Santa Catarina essa fitofisionomia possui apenas $26 \%$ da cobertura original, distribuída em áreas com menos de 50 ha (Sevegnani et al., 2013). Além da fragmentação, o corte seletivo de espécies com alto valor econômico, principalmente de araucária e imbuia, provocou alterações importantes nos remanescentes (Souza, 2007; Vibrans et al., 2012).

Poucos estudos foram desenvolvidos com a finalidade de avaliar o impacto antrópico sobre a Floresta Ombrófila Mista em Santa Catarina (Sampaio \& Guarino, 2007; Negrini et al., 2014). Neste sentido, o presente trabalho teve por objetivo verificar se a vegetação apresenta variações florísticas e estruturais consistentes em função da natureza da alteração, tendo por base fragmentos com históricos de uso e tempos de regeneração distintos. Ainda, verificar o status das populações de araucária (Araucaria angustifolia (Bertol.) Kuntze) e imbuia (Ocotea porosa (Nees \& Mart.) Barroso), historicamente, as duas espécies mais exploradas comercialmente.

\section{Material e métodos}

\section{Área de estudo}

As áreas estudadas estão inseridas nas bacias hidrográficas dos rios Chapecó, Irani e Jacutinga, na região meio-oeste do Estado de Santa Catarina, localizadas entre $26^{\circ} 50^{\prime}$ e $27^{\circ} 02^{\prime}$ S e $51^{\circ} 45^{\prime}$ e $51^{\circ} 57^{\prime} \mathrm{W}$, em altitudes que variam de 1.017 a $1.091 \mathrm{~m}$. O clima da região, segundo a classificação de Köppen, é do tipo $\mathrm{Cfb}$. Sua temperatura média anual varia entre $16^{\circ} \mathrm{C}$ e $18^{\circ} \mathrm{C}$, com médias mensais variando entre $11,5^{\circ} \mathrm{C}$, nos meses mais frios (junho e julho), e $20,7^{\circ} \mathrm{C}$, nos meses mais quentes (janeiro e fevereiro). A umidade relativa anual varia entre $76-80 \%$ e a precipitação média anual é de $1.815 \mathrm{~mm}$, bem distribuída ao longo do ano.

A geologia da região enquadra-se na formação Serra Geral. Essa formação é constituída por rochas vulcânicas basálticas de textura afanítica, amigdaloidal no topo dos derrames, com intercalações de arenitos intertrapeanos, e por efusivas ácidas e intermediárias, representadas por dacito/riodacito felsítico, riolitos felsíticos e pórfiros (Scheibe, 1986). Predominam, na região de estudo, três tipos de solos: Cambissolo Húmico, Nitossolo Háplico e Latossolo Bruno (Santos et al., 2013).

A região é composta, predominantemente, por mosaicos de vegetação nativa entremeada com plantios florestais de Pinus spp., e Eucaliptus spp. e, em menor escala, agricultura e pastagem. Especificamente nos fragmentos avaliados (pertencentes a uma empresa de celulose) tem em comum o entorno, formado por plantios florestais em diferentes idades. Assim, tem-se um mosaico de remanescentes florestais $(33 \%$ da cobertura da propriedade) em diferentes estados de conservação, em função do tempo e intensidade de uso pretérito. $\mathrm{O}$ histórico de impacto das áreas tem em comum o corte seletivo de espécies com valor econômico, em diferentes anos (Tabela 1). Além disso, em duas áreas, posterior ao corte seletivo, houve pastoreio e em outras duas a retirada de árvores de pequeno porte (lenha) do subbosque.

As informações relativas ao período em que ocorreu o impacto foram realizadas através de consulta aos documentos operacionais da empresa, onde contavam os relatórios de venda de madeira (mediante Planos de Manejo Sustentável) de cada fazenda. Posteriormente, foi realizada consulta a fotos antigas junto ao Centro Cultural da Celulose Irani (proprietária da área) que detém um acervo fotográfico desde a sua fundação, 
em 1940. De forma complementar, foram realizadas entrevistas com dois funcionários que atuavam na empresa há mais de 20 anos, que relataram parte do histórico de usos e ocupações desses locais, as quais foram comparadas com as duas fontes citadas anteriormente.

Tabela 1. Relação dos fragmentos de Floresta Ombrófila Mista analisados com seus respectivos históricos de uso e tempo de regeneração.

\begin{tabular}{ccc}
\hline Fragmentos & Impacto antrópico & $\begin{array}{c}\text { Tempo de } \\
\text { regeneração (anos) }\end{array}$ \\
\hline CL20_1 & Corte seletivo + lenha & 20 \\
CL20_2 & Corte seletivo + lenha & 20 \\
C20_1 & Corte seletivo & 20 \\
C20_2 & Corte seletivo & 20 \\
CP30_1 & Corte seletivo + pastoreio & 30 \\
CP30_2 & Corte seletivo + pastoreio & 30 \\
C20_3 & Corte seletivo & 20 \\
C20_4 & Corte seletivo & 20 \\
C40_1 & Corte seletivo & 40 \\
C40_2 & Corte seletivo & 40 \\
C40_3 & Corte seletivo & 40 \\
C40_4 & Corte seletivo & 40 \\
\hline
\end{tabular}

\section{Coleta dos dados}

A amostragem da vegetação foi realizada pelo método de parcelas (Müeller-Dombois \& Ellenberg, 2002), em 2009. Em cada área de estudo foram estabelecidas duas parcelas retangulares de $100 \mathrm{~m}$ x $20 \mathrm{~m}\left(2.000 \mathrm{~m}^{2}\right)$, subdivididas em subparcelas menores com $10 \mathrm{~m}$ x $20 \mathrm{~m}$ $\left(200 \mathrm{~m}^{2}\right)$. Para o levantamento do estrato arbóreo adulto foram amostrados os indivíduos vivos com perímetro à altura do peito $(\mathrm{PAP}) \geq 15 \mathrm{~cm}$. No interior de cada subparcela foi implantada uma parcela de $2 \mathrm{~m}$ x $2 \mathrm{~m}$ $\left(4 \mathrm{~m}^{2}\right)$, destinada ao levantamento da regeneração (juvenil), caracterizada pelos indivíduos com altura $\geq$ $20 \mathrm{~cm}$ e $\mathrm{PAP}<15 \mathrm{~cm}$.

O material botânico coletado foi identificado por comparação com exsicatas depositadas no Museu Botânico de Curitiba (MBM), Herbário Fernando Cardoso da Silva (HFC), da Embrapa Florestas, e mediante consulta à literatura taxonômica e aos especialistas da Universidade Federal do Paraná e do MBM.

\section{Análise dos dados}

Para detectar agrupamentos florísticos, formados pelos fragmentos que mais se assemelham, foram utilizadas duas matrizes de dados, uma constituída pela composição de espécies e a outra pela abundância de indivíduos das espécies. Na sequência, foi realizada uma ordenação do tipo escalonamento multidimensional não-métrico (NMDS), com intuito de investigar como os fragmentos se ordenavam no espaço multidimensional da composição e abundância das espécies. Nesta análise, utilizou-se a distância de Sørensen para a matriz de composição, recomendável para dados qualitativos, e a distância de Bray-Curtis para a matriz de abundância, indicado para dados quantitativos (Legendre \& Legendre, 2012). Para verificar a consistência do NMDS, calculouse uma medida de stress, que indica a proporção da variância das disparidades não explicadas pelo modelo NMDS (McCune \& Grace, 2002). A significância estatística do stress foi verificada por meio do teste de Monte Carlo com 999 permutações, e a confirmação da estabilidade do stress na porção final das iterações foi verificada, conforme sugerido por McCune \& Grace (2002). Por fim, testou-se a significância estatística dos grupos florísticos formados pelo NMDS por meio da ANOSIM (Clarke, 1993).

Com a finalidade de comparar a riqueza de espécies dos agrupamentos florísticos detectados, foram construídas curvas de rarefação pelo método Mao Tau (Colwell et al., 2004). Nesta análise, foi realizada a rarefação com base no número de indivíduos observados, pois comparações entre a riqueza de espécies de fragmentos diferentes podem ser influenciadas pela densidade de indivíduos de cada fragmento (Gotelli \& Colwell, 2001).

A estrutura da floresta dos agrupamentos florísticos foi comparada por meio dos atributos dendrométricos medidos: diâmetro à altura do peito (DAP) mínimo, médio, máximo e a sua variância; área basal total; número de indivíduos; número de fustes; número de indivíduos da regeneração; número de indivíduos de Araucaria angustifolia (araucária) e Ocotea porosa (imbuia) para comunidade adulta e juvenil. A comparação entre as variáveis dendrométricas dos grupos foi realizada por meio do teste $F$ e posterior teste $t$ para duas amostras, ambos utilizando um nível de significância de 5\% (Zar, 2010). Para verificar as espécies preferenciais dos grupos florísticos detectados, foram analisadas as espécies indicadoras e obtidos os valores indicadores para cada espécie, utilizando-se o método de Tichý \& Chytrý (2006). A significância estatística de cada valor indicador foi verificada por meio do teste de Monte Carlo com 999 permutações.

Pesq. flor. bras., Colombo, v. 36, n. 87, p.277-287, jul./set. 2016 


\section{Resultados e discussão}

A avaliação dos fragmentos submetidos a distúrbios antrópicos de diferentes intensidades e natureza identificou fortes diferenciações florísticas e estruturais na vegetação. No escalonamento multidimensional não-métrico (NMDS), a composição e a abundância de espécies nos fragmentos diferiram conforme o histórico de uso (Figura 1). Os dois primeiros eixos de ordenação das matrizes de composição e abundância segregaram os fragmentos com menor tempo de sucessão desde o distúrbio e com mais de um tipo de impacto antrópico, resultando, respectivamente, em $85 \%$ e $84 \%$ da correlação entre as distâncias de ordenação e o espaço n-dimensional original. $\mathrm{O}$ stress médio obtido para os dados reais (composição $=13,3$; abundância $=15,4)$ e aleatorizados $($ composição $=19,3$; abundância $=20,7)$ dos dois primeiros eixos de ordenação do NMDS se mantiveram estáveis na porção final das iterações, e apresentaram significância estatística pelo teste de Monte Carlo $(P<0,01)$. O conjunto de parcelas do grupo 1 é formado por sítios com maior tempo de intervenção e essa ficou restrita ao corte seletivo. O grupo 2 é formado por sítios com menor tempo de sucessão e por submetidos a impactos adicionais (Tabela 1).

a)

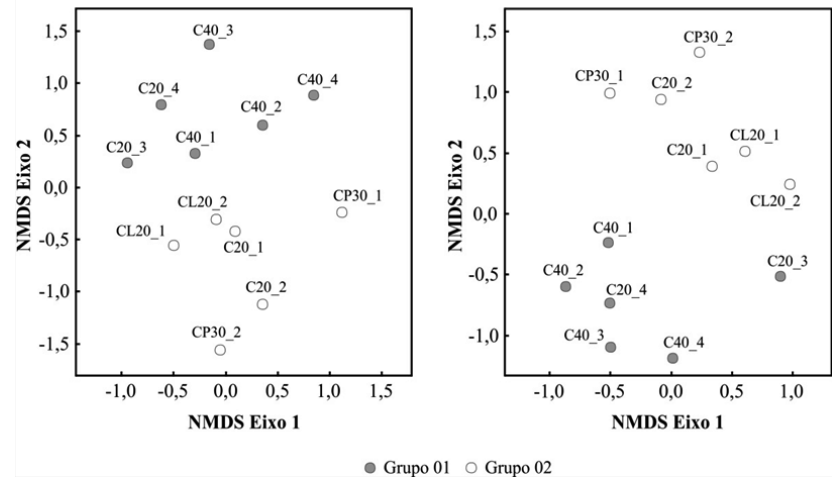

Figura 1. Diagramas de ordenação das parcelas produzidos pelo escalonamento multidimensional não-métrico (NMDS), com base na composição (a) e abundância (b) de espécies dos 12 fragmentos de Floresta Ombrófila Mista avaliados.

Em florestas subtropicais, a estrutura da vegetação tende a apresentar variações pronunciadas quando submetida a diferentes intensidades de distúrbios antrópicos (Chazdon, 2003; Souza, 2007; Souza et al., 2012). Fragmentos de Floresta Ombrófila Mista submetidos ao corte seletivo podem recuperar suas variáveis estruturais (área basal e densidade) em poucas décadas. Porém, a composição florística apresenta diferença acentuada, mesmo após 50 anos de sucessão (Souza et al., 2012). Assim, a manutenção da diferenciação florística observada entre os fragmentos, passados 20 e 40 anos, permite inferir que os efeitos de tais impactos sobre a riqueza podem perdurar por muitos anos, como observado em florestas tropicais (OsazuwaPeters et al., 2015).

A presença do fator impactante por um longo tempo (gado e corte de indivíduos de pequeno porte) pode afetar significativamente a comunidade arbórea, uma vez que limita a regeneração natural e o ingresso de novos indivíduos adultos (Sampaio \& Guarino, 2007; Vibrans et al., 2008; 2011). Esses fatores, atualmente, ainda estão muito presentes nas florestas catarinenses. Vibrans et al. (2011), por exemplo, avaliando 92 fragmentos de Floresta Ombrófila Mista em Santa Catarina, apontaram que $78 \%$ dos fragmentos foram submetidos ao corte seletivo de espécies com valor econômico e 54\% com uso para pastagens. Gasper et al. (2011), num total de 225 unidades amostrais, encontraram $68 \%$ dos fragmentos com corte seletivo e $56 \%$ com pastejo interno.

Para o estrato arbóreo adulto, foram amostrados 3.284 indivíduos, distribuídos em 99 espécies (Tabela 2). O Grupo 1 apresentou 76 espécies (24 exclusivas), e o Grupo 2 apresentou 75 espécies (23 exclusivas). Para o estrato juvenil, foram amostrados 335 indivíduos distribuídos em 76 espécies, sendo 45 espécies no conjunto de fragmentos do Grupo 1 e 53 do Grupo 2.

Os fragmentos com impactos mais antigos (Grupo 1) apresentaram maior abundância de espécies típicas de sub-bosque, como Rudgea jasminoides, Ilex paraguariensis, Dicksonia sellowiana e Myrceugenia sp. Aliado a isto, algumas espécies aparecem como indicadores desse conjunto de fragmentos (Tabela 3). Nesses, a presença de indivíduos formando um estrato inferior pode estar relacionado ao avanço da sucessão e ao estado de conservação (Liebsch et al., 2008), uma vez que espécies típicas do estrato inferior da floresta (por exemplo, rubiáceas e monimiáceas) são mais susceptíveis a impactos e à fragmentação. Nesses casos, essas espécies podem apresentar taxas de mortalidade pronunciadas, não sendo compensadas pelo recrutamento (Turner et al., 1996; Zhu et al., 2004). Os resultados convergem para uma acentuada diferenciação florística entre os fragmentos com diferentes intensidades de uso (Souza et al., 2012; Osazuwa-Peters et al., 2015). 
Tabela 2. Abundância de espécies arbóreas e atributos funcionais nos doze fragmentos de Floresta Ombrófila Mista analisados.

\begin{tabular}{|c|c|c|c|c|c|}
\hline \multirow{2}{*}{ Família } & \multirow{2}{*}{ Espécie } & \multicolumn{2}{|c|}{ Grupo 01} & \multicolumn{2}{|c|}{ Grupo 02} \\
\hline & & A & $\mathbf{J}$ & A & $\mathbf{J}$ \\
\hline \multirow[t]{2}{*}{ Acanthaceae } & Justicia carnea Lindl. & & 4 & & 4 \\
\hline & Justicia floribunda (C. Koch) Wassh. & & 12 & & \\
\hline Adoxaceae & Sambucus australis Cham. \& Schltdl. & 2 & & & \\
\hline \multirow[t]{2}{*}{ Anacardiaceae } & Lithraea brasiliensis Marchand & & & 84 & \\
\hline & Schinus terebintifolius Raddi & & & 12 & \\
\hline Annonaceae & Annona emarginata (Schltdl.) H.Rainer & 13 & 2 & 7 & 4 \\
\hline Apocynaceae & Aspidosperma pyricollum Müll.Arg. & 7 & & & \\
\hline \multirow[t]{4}{*}{ Aquifoliaceae } & Ilex dumosa Reissek & 16 & & 31 & 2 \\
\hline & Ilex paraguariensis A. St.-Hil. & 54 & & 6 & 2 \\
\hline & Ilex theezans Mart. ex Reissek & 4 & & 22 & \\
\hline & Ilex sp. & & 1 & & \\
\hline Araucariaceae & Araucaria angustifolia (Bertol.) Kuntze & 12 & 2 & 84 & 6 \\
\hline Asparagaceae & Cordyline spectabilis Kunth \& Bouché & 2 & & 8 & \\
\hline \multirow[t]{9}{*}{ Asteraceae } & Baccharis dentata (Vell.) Barroso & 1 & & & 1 \\
\hline & Baccharis oblongifolia (Ruiz \& Pav.) Pers. & 1 & & & \\
\hline & Dasyphyllum tomentosum (Spreng.) Cabrera & & & 22 & \\
\hline & Eupatorium sp. & & & & 1 \\
\hline & Moquiniastrum polymorphum (Less.) G. Sancho & 1 & & & \\
\hline & Piptocarpha angustifolia Dusén & 6 & & & \\
\hline & Vernonanthura discolor (Spreng.) H. Rob & 3 & & 5 & \\
\hline & Vernonanthura westiniana (Less.) H. Rob & 1 & & & \\
\hline & Vernonanthura sp. & & 1 & & \\
\hline Bignoniaceae & Jacaranda puberula Cham. & 4 & & 28 & \\
\hline Canellaceae & Cinnamodendron dinisii Schwacke & 7 & & 75 & 3 \\
\hline Cannabaceae & Celtis iguanaea (Jacq.) Sarg. & 5 & 2 & & \\
\hline Cardiopteridaceae & Citronella gongonha (Mart.) R. A. Howard & 3 & & & \\
\hline \multirow[t]{2}{*}{ Celastraceae } & Maytenus glaucescens Reissek & & & 11 & \\
\hline & Maytenus schumanniana Loes. & & & 6 & \\
\hline Clethraceae & Clethra scabra Pers. & 14 & & 90 & \\
\hline \multirow[t]{2}{*}{ Cunoniaceae } & Lamanonia ternata Vell. & 23 & & 121 & 1 \\
\hline & Weinmannia paulliniifolia Pohl ex Ser. & 4 & & 6 & \\
\hline Dicksoniaceae & Dicksonia sellowianaI Hook. & 92 & & 24 & \\
\hline Elaeocarpaceae & Sloanea monosperma Vell. & 63 & 1 & 14 & 1 \\
\hline Erythroxylaceae & Erythroxylum deciduum A. St-Hil. & & & 3 & \\
\hline \multirow[t]{4}{*}{ Euphorbiaceae } & Bernardia pulchella (Baill.) Müll. Arg. & & & & 4 \\
\hline & Gymnanthes klotzschiana Múll.Arg. & 25 & 5 & 28 & 3 \\
\hline & Sapium glandulosum (L.) Morong & 21 & & 16 & \\
\hline & Sebastiania brasiliensis Spreng. & & & 8 & 5 \\
\hline \multirow[t]{5}{*}{ Fabaceae } & Dahlstedtia muehlbergiana (Hass1.) M. J. Silva \& A. M. G. Azevedo & 28 & & & \\
\hline & Inga lentiscifolia Benth. & 3 & & 11 & \\
\hline & Inga striata Benth. & 7 & 1 & & \\
\hline & Mimosa scabrella Benth. & 12 & & 1 & \\
\hline & NI & & & & \\
\hline
\end{tabular}


Tabela 2. Continuação.

\begin{tabular}{|c|c|c|c|c|c|}
\hline \multirow{2}{*}{ Família } & \multirow{2}{*}{ Espécie } & \multicolumn{2}{|c|}{ Grupo 01} & \multicolumn{2}{|c|}{ Grupo 02} \\
\hline & & $\mathbf{A}$ & $\mathbf{J}$ & $\mathbf{A}$ & $\mathbf{J}$ \\
\hline Lamiaceae & Vitex megapotamica (Spreng.) Moldenke & 7 & & & \\
\hline \multirow[t]{7}{*}{ Lauraceae } & Nectandra grandiflora Nees. & 61 & 20 & & \\
\hline & Nectandra lanceolata Nees. & 7 & & 2 & \\
\hline & Nectandra megapotamica (Spreng.) Mez. & 14 & 8 & 18 & 1 \\
\hline & Ocotea porosa (Ness. \& Mart.) Barroso & 25 & 2 & 55 & 1 \\
\hline & Ocotea puberula (Rich.) Nees. & 1 & & 3 & 2 \\
\hline & Ocotea pulchella Mart. & 8 & 1 & 102 & 10 \\
\hline & Ocotea sp. & & & & 1 \\
\hline Loganiaceae & Strychnos brasiliensis Mart. & 3 & 1 & & \\
\hline \multirow[t]{4}{*}{ Melastomataceae } & Leandra carassana (DC.) Cogn. & & 2 & & 1 \\
\hline & Leandra hirtella Cogn. & & & & 1 \\
\hline & Leandra laevigata (Triana) Cogn. & & 1 & & \\
\hline & Miconia cinerascens Miq. & & 5 & 3 & 16 \\
\hline \multirow[t]{2}{*}{ Meliaceae } & Cabralea canjerana (Vell.) Mart. & 2 & & & \\
\hline & Cedrela fissilis Vell. & 3 & & & \\
\hline Moraceae & Sorocea bonplandii (Baill.) W. C. Burger et al. & & & 1 & \\
\hline \multirow[t]{27}{*}{ Myrtaceae } & Acca sellowiana (O. Berg) Burret & & & 4 & 1 \\
\hline & Calyptranthes concinna DC. & 19 & & 47 & \\
\hline & Campomanesia guazumifolia (Cambess.) O. Berg & 1 & & & \\
\hline & Campomanesia xanthocarpa $\mathrm{O}$. Berg & 34 & 4 & 15 & 2 \\
\hline & Eugenia handroana D. Legrand & 12 & & 1 & \\
\hline & Eugenia involucrata DC. & & & 6 & 1 \\
\hline & Eugenia pluriflora DC. & 5 & & 11 & \\
\hline & Eugenia pyriformis Cambess. & 5 & & & \\
\hline & Eugenia speciosa Cambess. & & & 15 & 3 \\
\hline & Eugenia uniflora L. & 7 & & 11 & \\
\hline & Eugenia sp. & & 3 & & \\
\hline & Myrceugenia euosma (O. Berg) D. Legrand & & & & 2 \\
\hline & Myrceugenia mesomischa (Burret) D. Legrand \& Kausel & 1 & & 1 & \\
\hline & Myrceugenia miersiana (Gardner) D. Legrand \& Kausel & 9 & 2 & 5 & \\
\hline & Myrceugenia myrcioides (Cambess.) O. Berg & 5 & 1 & 11 & 1 \\
\hline & Myrceugenia sp. & 93 & & 3 & \\
\hline & Myrcia guianensis (Aubl.) DC. & 13 & 1 & 93 & 1 \\
\hline & Myrcia oblongata DC. & & & 37 & 8 \\
\hline & Myrcia selloi (Spreng.) N. Silveira & 1 & & 9 & \\
\hline & Myrcia sp. & & 4 & & \\
\hline & Myrcianthes gigantea (D.Legrand) D. Legrand & 2 & & 3 & 1 \\
\hline & Myrcianthes pungens (O. Berg) D. Legrand & & & 1 & \\
\hline & Myrciaria floribunda (H. West ex Willd.) O. Berg & 16 & 4 & 7 & \\
\hline & Myrciaria tenella (DC.) O. Berg & & 1 & 7 & 3 \\
\hline & Myrrhinium atropurpureum Schott & 1 & & 1 & \\
\hline & Siphoneugena reitzii D. Legrand & 9 & & 5 & \\
\hline & NI 1 & & & & 2 \\
\hline
\end{tabular}


Tabela 2. Continuação.

\begin{tabular}{|c|c|c|c|c|c|}
\hline \multirow{2}{*}{ Família } & \multirow{2}{*}{ Espécie } & \multicolumn{2}{|c|}{ Grupo 01} & \multicolumn{2}{|c|}{ Grupo 02} \\
\hline & & $\mathbf{A}$ & $\mathbf{J}$ & $\mathbf{A}$ & $\mathbf{J}$ \\
\hline & NI 2 & & & & 1 \\
\hline & NI 3 & & & & 1 \\
\hline & NI 4 & & 1 & & \\
\hline & NI 5 & & 3 & & \\
\hline & NI 6 & & & & 2 \\
\hline & NI 7 & & 2 & & \\
\hline Piperaceae & Piper mikanianum (Kunth) Steud. & & 3 & & 5 \\
\hline Podocarpaceae & Podocarpus lambertii Klotzsch ex Endl. & & & 23 & \\
\hline \multirow[t]{2}{*}{ Primulaceae } & Myrsine coriacea (Sw.) Roem. \& Schult. & 1 & & 3 & 6 \\
\hline & Myrsine umbellata Mart. & 5 & 1 & & \\
\hline Proteaceae & Roupala montana var. brasiliensis (Klotzsch) K. S. Edwards & & & 3 & \\
\hline Quillajaceae & Quillaja brasiliensis (A. St.-Hil. \& Tul.) Mart. & & & 1 & \\
\hline Rhamnaceae & Scutia buxifolia Reissek & & & 6 & \\
\hline Rosaceae & Prunus myrtifolia (L.) Urb. & 12 & 4 & 15 & \\
\hline \multirow[t]{5}{*}{ Rubiaceae } & Cordiera concolor (Cham.) Kuntze & 2 & & & 1 \\
\hline & Palicourea australis C. M. Taylor & & 4 & & \\
\hline & Psychotria suterella Müll. Arg. & & 3 & & \\
\hline & Rudgea jasminoides (Cham.) Müll. Arg. & 101 & 14 & & \\
\hline & Rudgea parquioides (Cham.) Müll. Arg. & & & & 3 \\
\hline \multirow[t]{3}{*}{ Rutaceae } & Zanthoxylum rhoifolium Lam. & & 1 & 11 & 2 \\
\hline & Citrus sp. & & & 2 & \\
\hline & NI & & 1 & & \\
\hline Sabiaceae & Meliosma sellowii Urb. & 13 & 2 & 27 & 3 \\
\hline \multirow[t]{4}{*}{ Salicaceae } & Banara tomentosa Clos & 15 & & 3 & \\
\hline & Casearia decandra Jacq. & 64 & 4 & 45 & 8 \\
\hline & Casearia obliqua Spreng. & 5 & & 6 & \\
\hline & Xylosma pseudosalzmanii Sleumer & 1 & & 24 & 4 \\
\hline \multirow[t]{4}{*}{ Sapindaceae } & Allophylus edulis (A. St.-Hil. et al.) Hieron. ex Niederl. & 42 & 4 & 124 & 2 \\
\hline & Allophylus guaraniticus (A. St.-Hil.) Radlk. & 5 & & 3 & 3 \\
\hline & Cupania vernalis Cambess. & 97 & 6 & 66 & \\
\hline & Matayba elaeagnoides Radlk. & 43 & 12 & 330 & 16 \\
\hline \multirow[t]{4}{*}{ Solanaceae } & Cestrum intermedium Sendtn. & 3 & & 1 & \\
\hline & Solanum paranense Dusén & & 2 & & 1 \\
\hline & Solanum reflexum Schrank & & & & 1 \\
\hline & Solanum sanctae-catharinae Dunal & 5 & & & \\
\hline Styracaceae & Styrax leprosus Hook \& Arn. & 24 & 6 & 103 & 1 \\
\hline \multirow[t]{3}{*}{ Symplocaceae } & Symplocos tetrandra Mart. & & & 9 & \\
\hline & Symplocos uniflora (Pohl) Benth. & & & 17 & 6 \\
\hline & Symplocos sp. & & & & 2 \\
\hline Thymelaeaceae & Daphnopsis racemosa Griseb. & 1 & 3 & & \\
\hline \multirow[t]{2}{*}{ Winteraceae } & Drimys brasiliensis Miers & 1 & & 23 & 1 \\
\hline & NI & 1 & & & \\
\hline
\end{tabular}

$\mathrm{A}=$ adulto, $\mathrm{J}=$ juvenil. 
Tabela 3. Espécies indicadoras dos fragmentos em cada grupo de sítios segregados.

\begin{tabular}{lcc}
\hline \multicolumn{1}{c}{ Espécies indicadoras } & VI (\%) & $P$ \\
\hline Grupo 1 & 84,5 & 0,015 \\
Myrcengenia sp. & 84,5 & 0,016 \\
Piptocarpha angustifolia & 84,5 & 0,017 \\
Dahlstedtia muehlbergiana & 84,5 & 0,020 \\
Nectandra grandiflora & 70,7 & 0,049 \\
Eugenia pyriformis & 70,7 & 0,049 \\
Rudgea jasminoides & & \\
Grupo 2 & 100,0 & 0,005 \\
Lithraea brasiliensis & 100,0 & 0,005 \\
Maytenus glaucescens & 70,7 & 0,049 \\
Symplocos uniflora & 70,7 & 0,049 \\
Scutia buxifolia &
\end{tabular}

$\mathrm{VI}=$ valor indicador (significativo para $P \leq 0,05$ ).
Considerando a padronização de 1.238 indivíduos amostrados no estrato adulto, as curvas de rarefação indicaram que o conjunto que reúne os fragmentos com menor antropismo apresentou maior riqueza (76 espécies), quando comparado aos fragmentos mais antropizados (70 espécies) (Figura 2a). Entretanto, no estrato juvenil verificou-se que fragmentos mais antropizados apresentam maior riqueza de espécies (Figura 2b) e menor densidade total $(t=2,907 ; P<$ $0,05)$. Esse resultado indica que a riqueza de espécies da regeneração natural está intimamente relacionada à intensidade de distúrbio na floresta, em razão de fragmentos mais antropizados apresentarem maior heterogeneidade ambiental e, consequentemente, maior disponibilidade de espaço e recursos para a colonização de novas espécies (Gris et al., 2014). a)

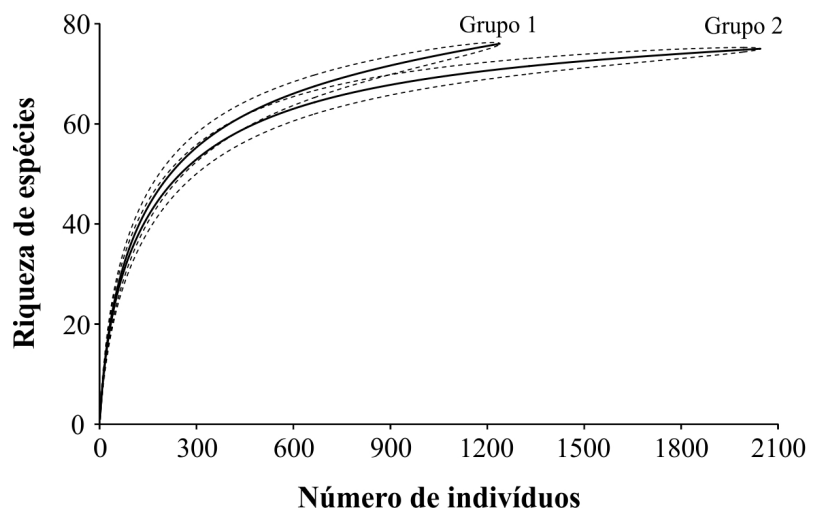

b)

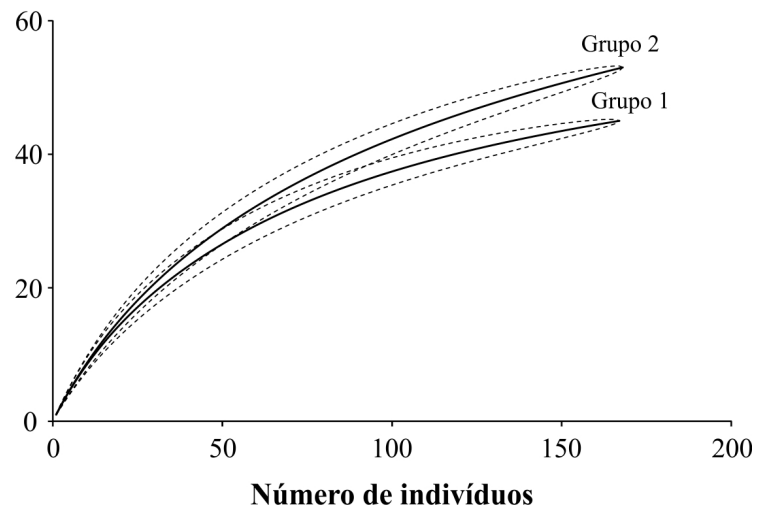

Figura 2. Curvas de rarefação obtidas pelo método Mao Tau, com seus respectivos intervalos de confiança ( \pm IC 95\%), para espécies arbóreas adultas (a) e juvenis (b) de fragmentos de Floresta Ombrófila Mista em dois grupos segregados.

Em relação à área basal, não houve diferenças significativas entre grupos $(P>0,05)$. Entretanto, foram observadas diferenças significativas para o DAP médio $(P<0,05)$ e a variância do DAP $(P<0,05)$, que foram maiores nas áreas do grupo 1 . A densidade de indivíduos foi maior nos sítios do grupo $2(P<0,001)$, onde se observou um acréscimo de $32 \%$ de indivíduos em relação ao grupo 1 (Tabela 3). Possivelmente, a semelhança entre a área basal ocorreu por haver, nas áreas mais jovens (grupo 2), um grande número de indivíduos de menor porte, sendo compensados por uma menor densidade de indivíduos de maior diâmetro, presentes nos fragmentos do grupo 1 (Denslow, 1995; Osazuwa-Peters et al., 2015). Entre as variáveis estruturais, a densidade de indivíduos arbóreos tem sido apontada como um indicador do estado de conservação para diferentes paisagens (Souza et al., 2012; Pereira et al., 2015). De fato, remanescentes em estádios iniciais de sucessão, ou mesmo naqueles locais com distúrbios recentes, apresentam uma maior densidade de indivíduos arbóreos, porém, com diâmetros menores (Denslow, 1995).

A maior frequência de múltiplos fustes foi encontrada nas áreas do grupo 2, aquelas com histórico de supressão de indivíduos do sub-bosque. A presença de múltiplos fustes é um indicativo de que houve corte de indivíduos (Simões et al., 2007). Antes do advento do gás de cozinha, a lenha era a principal fonte de energia para muitas famílias, sendo muito comum a retirada de 
pequenas árvores do sub-bosque para esse fim (Carvalho, 2011). Mesmo este hábito estando presente atualmente em menor escala, estudos recentes indicam que populações em comunidades rurais chegam a consumir de 680 a $980 \mathrm{~kg}$ de lenha por pessoa em um ano, sendo que esse combustível representa a segunda principal matriz energética do setor residencial no país, podendo resultar em significativas alterações na estrutura dos fragmentos (Ribeiro et al., 2015).

Para o estrato adulto, a densidade e a área basal de araucária foram estatisticamente maiores nas florestas do grupo $2(P<0,001)$. No entanto, para imbuia, tanto a densidade como a área basal não apresentaram diferenças significativas entre os dois conjuntos de fragmentos $(P$ $>0,05)$. No estrato juvenil, para ambas as espécies, a densidade é extremamente baixa e não diferiram entre os fragmentos $(P>0,05)$ (Tabela 3$)$. Essas espécies, que outrora ocupavam importância fisionômica marcante na Floresta Ombrófila Mista na região centro-oeste de Santa Catarina (Klein, 1978), atualmente encontramse descaracterizadas (Vibrans et al., 2008; Souza et al., 2012; Sevegnani et al., 2013), por serem as duas espécies com maior intensidade de exploração (Caldato et al., 1999). Em condições naturais, sem interferência antrópica, Araucaria angustifolia formava um dossel homogêneo e contínuo, com sub-dossel composto frequentemente por Ocotea porosa (Klein, 1978).

A atual densidade de araucária nos fragmentos estudados está abaixo da esperada para o componente adulto (10 a 70 ind $\left.\mathrm{ha}^{-1}\right)$ e extremamente baixo no juvenil (1,7 a 5 ind $\left.\mathrm{ha}^{-1}\right)$. Para indivíduos adultos, a densidade média dos fragmentos em Santa Catarina é de 75 ind ha ${ }^{-1}$ (Vibrans et al., 2008) e no Paraná os valores variam de 116 a 166 ind ha-1 (Sanquetta et al., 2003). Registros históricos de florestas com pouca intervenção apontam para densidade de 200 ind ha $^{-1}$ (Klein, 1978). No estrato juvenil, Paludo et al. (2011) encontraram uma densidade de 48 ind ha ${ }^{-1}$ na Floresta Nacional de Caçador, SC. No Parque Nacional do Iguaçu, a maior Unidade de Conservação de Proteção Integral do Paraná, foi registrada uma densidade de 208 ind ha-1 adultos e 160 ind ha-1 juvenis (Albiero Junior et al., 2015).

Em relação à imbuia, o estrato juvenil apontou valores baixos para os dois conjuntos de fragmentos (6,7 e 1,7 ind ha ${ }^{-1}$, para os grupos 1 e 2, respectivamente). Esses valores são muito inferiores aos registrados em cinco fragmentos no interior do Paraná, onde os valores variaram entre 60 a 440 ind ha ${ }^{-1}$ (Munhoz et al., 2014), ou em Santa Catarina, na Reserva Genética Florestal de Caçador, onde foram encontrados 39 ind ha-1 (Caldato et al., 1999). Assim, é possível inferir que o histórico de uso é um dos responsáveis pela baixa densidade populacional encontrada, uma vez que a densidade de indivíduos jovens é afetada pelo corte seletivo e pela fragmentação das florestas (Munhoz et al., 2014). De forma complementar, a baixa área basal dessas espécies no estrato adulto deve ser consequência da intensa exploração (Tabela 4), já que em florestas pouco alteradas elas ocupavam os maiores valores de área basal entre todas as espécies (Vibrans et al., 2008).

Tabela 4. Parâmetros estruturais dos estratos arbóreos adulto e juvenil para fragmentos de Floresta Ombrófila Mista com baixo e alto impacto antrópico.

\begin{tabular}{|c|c|c|c|c|}
\hline \multirow[b]{2}{*}{ Parâmetro } & \multicolumn{4}{|c|}{ Impacto antrópico } \\
\hline & $\begin{array}{c}\text { Grupo } 1 \\
(n=60)\end{array}$ & $\begin{array}{c}\text { Grupo } 2 \\
(\mathrm{n}=60)\end{array}$ & $\mathbf{t}$ & $P$ \\
\hline \multicolumn{5}{|l|}{ Arbóreo adulto } \\
\hline Densidade (ind ha-1) & $1.009 \pm 101$ & $1.406 \pm 138,0$ & 4,647 & $<0,001$ \\
\hline $\begin{array}{l}\text { Caules bifurcados } \\
\left(\text { ind }^{-1} \mathrm{a}^{-1} \text { ) }\right.\end{array}$ & $33,3 \pm 19,0$ & $311,7 \pm 105,0$ & 5,194 & $<0,001$ \\
\hline Área basal $\left(\mathrm{m}^{2} \mathrm{ha}^{-1}\right)$ & $43,61 \pm 8,39$ & $45,64 \pm 7,00$ & 0,372 & 0,71 \\
\hline DAP médio $(\mathrm{cm})$ & $18,2 \pm 1,2$ & $14,8 \pm 0,8$ & 4,717 & $<0,001$ \\
\hline Variância DAP & $247,5 \pm 95,3$ & $130,5 \pm 23,0$ & 2,386 & 0,02 \\
\hline $\begin{array}{l}\text { Densidade araucária } \\
\left.\left(\text { ind }^{-1}\right)^{-1}\right)\end{array}$ & $10,0 \pm 7,0$ & $70,0 \pm 25,0$ & 4,648 & $<0,001$ \\
\hline $\begin{array}{l}\text { Densidade imbuia } \\
\left.\left(\text { ind }^{-1}\right)^{-1}\right)\end{array}$ & $19,2 \pm 9,0$ & $29,2 \pm 14,0$ & 1,197 & 0,23 \\
\hline $\begin{array}{l}\text { Dominância } \\
\text { araucária }\left(\mathrm{m}^{2} \mathrm{ha}^{-1}\right)\end{array}$ & $1,36 \pm 1,07$ & $8,82 \pm 3,38$ & 4,206 & $<0,001$ \\
\hline $\begin{array}{l}\text { Dominância imbuia } \\
\left(\mathrm{m}^{2} \mathrm{ha}^{-1}\right)\end{array}$ & $8,25 \pm 7,52$ & $4,21 \pm 2,82$ & 1,021 & 0,31 \\
\hline \multicolumn{5}{|l|}{ Arbóreo juvenil } \\
\hline Densidade (ind.ha ${ }^{-1}$ ) & $27.585 \pm 4.907$ & $18.833 \pm 3.491$ & 2,907 & 0,004 \\
\hline $\begin{array}{l}\text { Densidade araucária } \\
\text { (ind.ha }{ }^{-1} \text { ) }\end{array}$ & $1,7 \pm 2,3$ & $5,0 \pm 4,6$ & 1,298 & 0,19 \\
\hline $\begin{array}{l}\text { Densidade imbuia } \\
\text { (ind } \mathrm{ha}^{-1} \text { ) }\end{array}$ & $6,7 \pm 9,0$ & $1,7 \pm 2,3$ & 1,071 & 0,29 \\
\hline
\end{tabular}

\section{Conclusões}

Os impactos antrópicos, tais como corte seletivo de madeira para serraria ou lenha e o pastoreio no interior da floresta, foram tão profundos que, mesmo passadas algumas décadas de sucessão, as diferenças florísticas e estruturais são ainda evidentes.

As populações de araucária e de imbuia, as duas espécies historicamente mais exploradas da Floresta 
Ombrófila Mista, estão extremamente ameaçadas, pela baixa densidade de indivíduos adultos e de regenerantes nos fragmentos avaliados.

Como praticamente todos os fragmentos de Floresta Ombrófila Mista foram submetidos a algum tipo de intervenção antrópica, é importante considerar a estrutura populacional de espécies ameaçadas de extinção no planejamento de ações para conservação dos remanescentes da Floresta com Araucaria.

\section{Referências}

Albiero Junior, A. et al. Population structure of Araucaria angustifolia in the Iguaçu National Park. Floresta, v. 45, n. 2, p. 361-372, 2015. DOI: 10.5380/ rf.v45i2.35673.

Brasil. Lei $\mathbf{n}^{\mathbf{0}} \mathbf{1 1 . 4 2 8}$, de 22 de dezembro de 2006. Dispõe sobre a utilização e proteção da vegetação nativa do Bioma Mata Atlântica, e dá outras providências. Disponível em: <http://www.planalto.gov.br/ccivil_03/_ ato2004-2006/2006/lei/111428.htm>. Acesso em: 6 ago. 2015.

Caldato, S. L. et al. Estrutura populacional de Ocotea porosa (Lauraceae) em uma Floresta Ombrófila Mista, em Caçador (SC). Ciência Florestal, v. 9, n. 1, p. 89-101, 1999.

Carvalho, M. M. X. Os fatores do desmatamento da floresta com araucária: agropecuária, lenha e indústria madeireira. Revista Esboços, v. 18, n. 25, p. 32-52, 2011. DOI: 10.5007/2175-7976.2011v18n25p32.

Chazdon, R. L. Tropical forest recovery: legacies of human impact and natural disturbances. Perspectives in Plant Ecology, Evolution and Systematics, v. 6, n. 1-2, p. 51-71, 2003. DOI: 10.1078/1433-8319-00042.

Clarke, K. R. Non-parametric multivariate analysis of changes in community structure. Australian Journal of Ecology, v. 18, n. 1, p. 117-143, 1993. DOI: 10.1111/j.1442-9993.1993.tb00438.x.

Colwell, R. K. et al. Interpolating, extrapolating, and comparing incidencebased species accumulation curves. Ecology, v. 85, n. 10, p. 2717-2727, 2004. DOI: $10.1890 / 03-0557$.

Denslow, J. S. Disturbance and diversity in tropical rain forests: the density effect. Ecological Applications, v. 5, n. 4, p. 962-968, 1995. DOI: $10.2307 / 2269347$

Duarte, L. D. S. et al. Phylobetadiversity among forest types in the Brazilian Atlantic Forest Complex. PLoS ONE, v. 9, n. 8, p. 1-10, 2014. DOI: 10.1371/ journal.pone.0105043.

Fearnside, P. M. Desmatamento na Amazônia: dinâmica, impactos e controle. Acta Amazonia, v. 36, n. 3, p. 395-400, 2006. DOI: 10.1590/S004459672006000300018 .

Gasper, A. L. et al. Inventário de Dicksonia sellowiana Hook. em Santa Catarina. Acta Botanica Brasilica, v. 25, n. 4, p. 776-784, 2011. DOI: 10.1590/S0102-33062011000400005.

Gasper, A. L. et al. Inventário florístico florestal de Santa Catarina: espécies da Floresta Ombrófila Mista. Rodriguésia, v. 64, n. 2, p. 201-210, 2013.

Gotelli, N. J. \& Colwell, R. K. Quantifying biodiversity: procedures and pitfalls in the measurement and comparison of species richness. Ecology Letters, v. 4, n. 4, p. 379-391, 2001.

Gris, D. et al. Structure and floristic diversity of remnant semideciduous forest under varying levels of disturbance. Acta Botanica Brasilica, v. 28, n. 4, p. 569-576, 2014. DOI: 10.1590/0102-33062014abb3432.

IBGE. Manual técnico da vegetação brasileira. 2 ed. Rio de Janeiro: IBGE, 2012. 274 p.
Karsten, R. J. et al. Regeneration in canopy gaps of tierra-firme forest in the Peruvian Amazon: comparing reduced impact logging and natural, unmanaged forests. Forest Ecology and Management, v. 310, n. 1, p. 663-671, 2013. DOI: $10.1016 /$ j.foreco.2013.09.006.

Klein, R. M. Mapa fitogeográfico do estado de Santa Catarina. In: Reitz, R. (Ed.). Flora ilustrada catarinense. Itajaí: Herbário Barbosa Rodrigues, 1978.

Legendre, P. \& Legendre, L. Numerical ecology. Amsterdam: Elsevier, 2012.

Liebsch, D. et al. How long does the Atlantic Rain Forest take to recover after a disturbance? Changes in species composition and ecological features in the secondary succession. Biological Conservation, v. 141, n. 6, p. $1717-$ $1725,2008$.

Mccune, B. \& Grace, J. B. Analysis of ecological communities. Gleneden Beach: MjM Software Desing, 2002.

Müeller-Dombois, D. \& Ellenberg, H. Aims and methods of vegetation ecology. New Jersey: The Blackburn Press, 2002.

Munhoz, C. A. et al. Demography of the endangered tree species Ocotea porosa (Lauraceae) along a gradient of forest disturbance in southern Brazil. Acta Botanica Brasilica, v. 28, n. 4, p. 617-623, 2014. DOI: 10.1590/0102$33062014 a b b 3516$

Negrini, M. et al. Heterogeneidade florístico-estrutural do componente arbóreo em um sistema de fragmentos florestais no Planalto Sul catarinense. Revista Árvore, v. 38, n. 5, p. 779-786, 2014. DOI: 10.1590/S010067622014000500002 .

Osazuwa-Peters, O. L. et al. Selective logging: do rates of forest turnover in stems, species composition and functional traits decrease with time since disturbance? - a 45 year perspective. Forest Ecology and Management, v. 357, n. 1, p. 10-21, 2015. DOI: 10.1016/j.foreco.2015.08.002.

Paludo, G. F. et al. Regeneração de uma população natural de Araucaria Angustifolia (Araucariaceae). Revista Árvore, v. 35, n. 5, p. 1107-1119, 2011. DOI: 10.1590/S0100-67622011000600017.

Pereira, J. A. A. et al. Human impacts affect tree community features of 20 forest fragments of a vanishing neotropical hotspot. Environmental Management, v. 55, n. 2, p. 296-307, 2015.

Ribeiro, E. M. S. et al. Chronic anthropogenic disturbance drives the biological impoverishment of the Brazilian Caatinga vegetation. Journal of Applied Ecology, v. 52, n. 3, p. 611-620, 2015. DOI: 10.1111/1365-2664.12420.

Ribeiro, M. C. et al. The Brazilian Atlantic Forest: how much is left, and how is the remaining for distributed? Implications for conservation. Biological Conservation, v. 142, n. 6, p. 1141-1153, 2009. DOI: 10.1016/j. biocon.2009.02.021.

Sampaio, M. B. \& Guarino, E. S. G. Efeitos do pastoreio de bovinos na estrutura populacional de plantas em fragmentos de floresta Ombrófila Mista. Revista Árvore, v. 31, n. 6, p. 1035-1046, 2007. DOI: 10.1590/ S0100-67622007000600008.

Sanquetta, C. R. et al. Crescimento, mortalidade e recrutamento em duas florestas de araucária (Araucaria angustifolia (Bert.) O. Ktze.) no estado do Paraná, Brasil. Revista Ciências Exatas e Naturais, v. 5, n. 1, p. 101-112, 2003.

Santos, H, G. et al. Sistema brasileiro de classificação de solos. 3 ed. Rio de Janeiro: Embrapa Solos, 2013.

Scheibe, L. F. A geologia de Santa Catarina: sinopse provisória. Geosul, v. 1, n. 1, p. $7-38,1986$.

Sevegnani, L. et al. Considerações finais sobre a Floresta Ombrófila Mista em Santa Catarina. In: Vibrans, A. C. et al. (Ed.). Inventário florístico florestal de Santa Catarina: Floresta Ombrófila Mista. Blumenau: EDIFURB, 2013.

Simões, C. G. \& Marques, M. C. M. The role of sprouts in the restoration of Atlantic Rainforest in southern Brazil. Restoration Ecology, v. 15, n. 1, p. 53-59, 2007. DOI: 10.1111/j.1526-100X.2006.00189.x. 
Souza, A. F. Ecological interpretation of multiple population size structures in trees: the case of Araucaria angustifolia in South America. Austral Ecology, v. 32, n. 5, p. 524-533, 2007. DOI: 10.1111/j.1442-9993.2007.01724.x.

Souza, A. F. et al. Native forest management in subtropical South America: long-term effects of logging and multiple-use on forest structure and diversity. Biodiversity and Conservation, v. 21, n. 8, p. 1953-1969, 2012. DOI: 10.1007/s10531-012-0287-1.

Tabarelli, M. \& Mantovani, W. A regeneração de uma Floresta Tropical Montana após corte e queima (São Paulo-Brasil). Revista Brasileira de Biologia, v. 59, n. 2, p. 239-250, 1999. DOI: 10.1590/S003471081999000200008 .

Tichý, L. \& Chytrý, M. Statistical determination of diagnostic species for site groups of unequal size. Journal of Vegetation Science, v. 17, n. 6, p. 809-818, 2006. DOI: 10.1111/j.1654-1103.2006.tb02504.x.

Turner, I. M. et al. A century of plant species loss from an isolated fragment of lowland tropical rain forest. Conservation Biology, v. 10, n. 4, p. 12291244, 1996.
Vibrans, A. C. et al. Extensão original e atual da cobertura florestas de Santa Catarina. In: Vibrans, A. C. et al. (Ed.). Inventário florístico florestal de Santa Catarina: diversidade e conservação dos remanescentes florestais. Blumenau: EDIFURB, 2012.

Vibrans, A. C. et al. Ordenação dos dados de estrutura da Floresta Ombrófila Mista partindo de informações do Inventário Florístico-Florestal de Santa Catarina: resultados de estudo-piloto. Ciência Florestal, v. 18, n. 4, p. 511523, 2008. DOI: $10.5902 / 19805098434$.

Vibrans, A. C. et al. Structure of mixed ombrophyllous forests with Araucaria angustifolia (Araucariaceae) under external stress in southern Brazil. Revista de Biología Tropical, v. 59, n. 3, p. 1371-1387, 2011. DOI: 10.15517/rbt. v0i0.3405.

Zar, J. H. Biostatistical analysis. New Jersey: Upper Saddle River, 2010.

Zhu, H. et al. Tropical rain forest fragmentation and its ecological and species diversity changes in southern Yunnan. Biodiversity and Conservation, v. 13, n. 7, p. 1355-1372, 2004. DOI: 10.1023/B:BIOC.0000019397.98407.c3. 
